\title{
$\mathrm{BMJ}$
}

\section{Lifestyle interventions for knee pain in overweight and obese adults aged $\geq 45$ : economic evaluation of randomised controlled trial}

\author{
Garry R Barton, senior lecturer in health economics, ${ }^{1}$ Tracey H Sach, senior lecturer in health economics, ${ }^{1,2,3}$ \\ Claire Jenkinson, research associate, ${ }^{2}$ Michael Doherty, professor of rheumatology, ${ }^{4}$ Anthony J Avery, \\ professor of primary care, ${ }^{2}$ Kenneth R Muir, professor of epidemiology ${ }^{2}$
}

${ }^{1}$ Health Economics Group, School of Medicine, Health Policy and

Practice, University of East Anglia, Norwich NR4 7T

${ }^{2}$ School of Community Health Sciences, University of

Nottingham, Nottingham NG7 2RD

${ }^{3}$ School of Pharmacy, University of East Anglia, Norwich

${ }^{4}$ Academic Rheumatology, University of Nottingham,

Nottingham

Correspondence to: T Sach, School of Pharmacy, University of East

Anglia, Norwich NR4 7TJ

T.Sach@uea.ac.uk

Cite this as: BMJ 2009;339:b2273 doi:10.1136/bmi.b2273

\section{ABSTRACT}

Objective To estimate the cost effectiveness of four different lifestyle interventions for knee pain.

Design Cost utility analysis of randomised controlled trial. Setting Five general practices in the United Kingdom. Participants 389 adults aged $\geq 45$ with self reported knee pain and body mass index (BMI) $\geq 28$.

Interventions Dietary intervention plus quadriceps strengthening exercises, dietary intervention, quadriceps strengthening exercises, and leaflet provision.

Participants received home visits over a two year period. Main outcome measure Incremental cost per quality adjusted life year (QALY) gained over two years from a health service perspective.

Results Advice leaflet was associated with a mean change in cost of $-£ 31$, and a mean QALY gain of 0.085 . Both strengthening exercises and dietary intervention were more effective (0.090 and 0.133 mean QALY gain, respectively) but were not cost effective. Dietary intervention plus strengthening exercises had a mean cost of $£ 647$ and a mean QALY gain of 0.147 and was estimated to have an incremental cost of $£ 10469$ per QALY gain (relative to leaflet provision), and a $23.1 \%$ probability of being cost effective at a $£ 20000 /$ ALY threshold.

Conclusion Dietary intervention plus strengthening exercises was estimated to be cost effective for individuals with knee pain, but with a large level of uncertainty.

Trial registration ISRCTN93206785.

\section{INTRODUCTION}

Nearly half of people aged $>50$ report having knee pain at some point in the past year, and, of these, a third report consulting their general practitioner with regard to their knee pain. ${ }^{1}$ Previous economic evaluations for people with knee pain have estimated only the cost effectiveness of different exercise programmes and have not focused on those who are overweight. ${ }^{23}$ The importance of such an evaluation was highlighted by a recent study that found that there was an early reliance on pharmacological treatments at the expense of non-pharmacological treatments such as weight loss, exercise, and the provision of written advice. ${ }^{4}$ This occurred despite the fact that these three treatments have been recommended as first line treatments for people with knee pain. ${ }^{5}$

A previous study evaluated a weight reduction programme ${ }^{6}$ as weight loss has been shown to be associated with an improvement in the symptoms of knee osteoarthritis, ${ }^{7}$ of which pain is the principal feature. ${ }^{8}$ Indeed, it has been estimated that between a quarter and a half of all knee osteoarthritis might be prevented by eliminating obesity. ${ }^{9}$ The value of a quadriceps strengthening exercise programme has also been estimated as there is evidence that quadriceps weakness is associated with disability and pain in patients with knee osteoarthritis ${ }^{1011}$ and that a home based exercise programme can help improve self reported knee pain and function. ${ }^{1213}$

We investigated the cost effectiveness of four interventions designed to alleviate knee pain in overweight and obese adults.

\section{METHODS}

Participants

The lifestyle interventions for knee pain study compared the effectiveness ${ }^{14}$ and cost effectiveness of four different intervention groups: dietary intervention plus quadriceps strengthening exercises, dietary intervention, quadriceps strengthening exercises, and leaflet provision. We sent an ascertainment questionnaire to all registered patients aged $\geq 45$ in five Nottingham general practices. We did not contact terminally ill patients and those with psychiatric illness, dementia, or any other incapacitating disease that their general practitioner thought made them unsuitable for participation. A local media campaign was also conducted to improve recruitment. Recruitment started in May 2003 and ended in March 2005. Responding individuals were recruited into the study if they reported that they had had knee pain on most days of the past month, had a body mass index $(\mathrm{BMI})>28.0$, and gave consent to be randomised to one of the four 
intervention groups (a greater proportion was randomised to dietary intervention because we anticipated a higher rate of attrition in this group).

\section{Interventions}

The interventions are described more fully in the accompanying clinical paper. ${ }^{14}$ Participants in both the dietary intervention groups were visited by a dietitian and received a personalised dietary plan that would create a deficit of $2.5 \mathrm{MJ}(600 \mathrm{kcal})$ a day. They were scheduled to receive visits every month in the first six months and every other month for the remainder of the 24 month intervention period. Participants in the exercise groups were taught exercises designed to strengthen the quadriceps (thigh) muscles and included resistance exercises, some of which were taught with rubber exercise (dyno) bands of varying strength. They were asked to repeat these exercises at home on a daily basis and were scheduled to receive visits every four months throughout the 24 month intervention period. The final intervention was the provision of a leaflet based on the Arthritis Research Campaign (ARC) Osteoarthritis of the Knee leaflet (www. arc.org.uk) but leaflets had all exercise and dietary advice removed (this was considered equivalent to standard care).

\section{Measuring costs}

For each participant we estimated the overall change in cost to the health service over the two year trial period by summing the costs associated with visits by healthcare professionals and the change in the costs associated with analgesic use. All costs were estimated in UK sterling (£) at 2006-7 financial year costs. Costs that occurred in the second year were discounted at the current recommended rate of 3.5\%. ${ }^{15}$

Visit costs - Throughout the study each healthcare professional recorded the number of visits to each participant. All visits were made to provide advice, with the exception of visits to the leaflet group, where visits were undertaken to record trial outcome information such as weight and the percentage of body fat. As such visits would not be made if the intervention was routinely provided in the NHS, we assumed that the visit cost for all participants who received the leaflet was equivalent to zero. The unit cost of all other visits was estimated from a previously published source ${ }^{16}$ and included the additional time and travel costs associated with home visits. The cost of providing dyno bands to participants who were allocated to either of the strengthening exercise groups was also estimated (www.physioroom.com).

Analgesic costs - At baseline, participants were asked to report whether they currently used analgesics (obtained by prescription or over the counter) and, if so, which type. The same question was also asked of participants at 12 and 24 months after randomisation. These data were combined with unit cost data ${ }^{17}$ to estimate the four weekly cost of analgesics. When data were missing, we used multiple imputation ${ }^{18}$ to generate replacement cost values for participants who did not provide information on analgesic use at a particular follow-up point. Subsequently, for each participant, we used the area under the curve method, ${ }^{19}$ with baseline adjustment, to estimate the change in analgesic costs, over the 24 month trial period. The mean change in analgesic costs could thereby be calculated for each intervention group, and this was added to the mean visit cost to estimate the mean overall change in cost to the health service for each of the four intervention groups.

Use of other resources-Additionally, to provide an indication of whether participants who received a particular intervention had a relatively high or low level of use of other health service resources, we asked a subset of participants (at one year after randomisation) how many visits related to knee pain (in the preceding four weeks) they had made to the general practitioner, nurse, and other healthcare professionals or hospital (outpatient visits and inpatient admissions were recorded). For practical reasons, the subsample consisted of 229 consecutive participants who had reached the one year follow-up between June 2004 and August 2005 . We used one way analysis of variance to assess whether there were significant differences in the mean number of visits across the four intervention groups.

\section{Measuring outcomes}

The accompanying clinical paper ${ }^{14}$ details the results according to the trial primary outcome measure of pain, as assessed by the Western Ontario and McMaster Universities osteoarthritis index (WOMAC). ${ }^{20}$ To estimate the impact that each intervention had on health related quality of life we asked participants to complete the EQ-5D ${ }^{21}$ at baseline and at six, 12 , and 24 months after randomisation. This enabled us to carry out a cost utility analysis, ${ }^{22}$ in which we compared the benefits of different healthcare interventions on a common scale (utility is measured on a scale where 0 is equivalent to death and 1 is equivalent to full health). A utility score was assigned to each of the health state descriptions elicited by the EQ-5D with the York A1 tariff, ${ }^{23}$ on which scores range between -0.594 and 1.00. After using multiple imputation ${ }^{18}$ to predict missing EQ-5D scores, we used the area under the curve method (with adjustment for baseline differences) ${ }^{19}$ to estimate, for each participant, the quality adjusted life year (QALY) gain/loss that accrued over the trial period. QALY gains that accrued in the second year were discounted at 3.5\%, and the mean QALY gain was estimated for each of the four intervention groups.

\section{Cost effectiveness}

The level of cost effectiveness was estimated from the viewpoint of the NHS. We excluded dominated interventions (which had a higher mean overall change in cost to the health service and lower mean QALY gain than another intervention) and interventions that were subject to extended dominance (as combinations of other interventions could provide a greater benefit at equivalent cost). ${ }^{24}$ Subsequently, we calculated the cost effectiveness of non-excluded interventions by 
estimating the incremental cost per QALY gain (incremental cost effectiveness ratio) associated with each intervention group, relative to the next best alternative. Confidence intervals for the incremental cost effectiveness ratio were also computed by using the bootstrap technique. ${ }^{25}$ In line with guidance provided by the National Institute of Health and Clinical Excellence $(\mathrm{NICE})^{26}$ we sought to identify the most cost effective intervention by comparing the incremental cost effectiveness ratio with a cost effectiveness threshold $(\lambda)$ of $£ 20000-£ 30000$ per QALY.

\section{Decision uncertainty}

To estimate the level of uncertainty associated with the decision as to which intervention was most cost effective we used probabilistic methods to estimate the cost effectiveness acceptability curve for each intervention group, where the curve depicts the probability that an intervention is cost effective at different levels of the cost effectiveness threshold. ${ }^{2728}$

\section{Sensitivity analysis}

One of our main assumptions was that all visits to those receiving the leaflet were undertaken solely to record trial outcome information and that these visits would not be made if the intervention was routinely provided in the NHS. Rather than assigning a zero cost to the visits received by those in the leaflet arm, in the sensitivity analysis we assumed that they should be assigned the same unit cost as those who received quadriceps strengthening exercises.

\section{RESULTS}

\section{Participants}

Questionnaires were returned by 8004 of the 12408 individuals $(65 \%)$ who were sent an ascertainment questionnaire. Of these, 320 were eligible to take part and consented to be randomised to one of the four interventions. An additional 69 participants were recruited via the media campaign. In total, 109 participants were randomised to receive dietary intervention plus quadriceps strengthening exercises, 122 to dietary intervention, 82 to quadriceps strengthening exercises, and 76 to leaflet provision. The mean age of the 389 participants was 61.3 and $257(66 \%)$ were women. According to BMI, 90 (23\%) were classified as overweight (BMI 25-<30), $196(50 \%)$ as class I obese (30$<35), 65(17 \%)$ as class II obese $(35-<40)$, and $38(10 \%)$ as class III obese $(\geq 40)$.

\section{Costs}

The mean number of visits received by participants randomised to dietary intervention plus quadriceps strengthening exercises was 11.19 (range 0-16), compared with 13.46 for dietary intervention alone (range 0-17), 4.95 for quadriceps strengthening exercises (range 0-7), and 6.05 (range 0-7) for those who received the leaflet (more participants withdrew from the exercise groups, which might explain why, on average, they received fewer visits than those who received a leaflet, see accompanying clinical paper for further information $^{14}$ ). The estimated unit cost per home visit was $£ 54.60$ for both the dietary intervention groups, and $£ 44.60$ for those who received the quadriceps strengthening exercises; the dyno bands cost $£ 5.50$. Over the two year trial period the estimated mean visit cost for those who received dietary intervention plus quadriceps strengthening exercises was $£ 615.64$ compared with $£ 735.57$ for the dietary intervention, $£ 214.66$ for the quadriceps strengthening exercises, and $£ 0.00$ for the leaflet (table 1 ).

At baseline, 12 months, and 24 months the associated question on analgesic costs was completed by an average of 287 participants (74\%). After imputation, for each of

Table 1 | Estimates of mean change in cost (£) and mean (SD) QALY gain associated with each intervention and their component parts

\begin{tabular}{|c|c|c|c|c|}
\hline & $\begin{array}{l}\text { Dietary intervention plus } \\
\text { strengthening exercises }\end{array}$ & Dietary intervention & Strengthening exercises & Leaflet provision \\
\hline \multicolumn{5}{|l|}{ Analgesic costs: } \\
\hline Baseline & $6.23(6.19)$ & $6.59(5.72)$ & $6.60(6.22)$ & $7.86(7.12)$ \\
\hline 12 month & $5.14(5.53)$ & $5.62(5.79)$ & $5.80(6.60)$ & $6.26(6.58)$ \\
\hline 24 month & $5.88(6.05)$ & $5.75(5.05)$ & $6.76(6.45)$ & $6.15(5.09)$ \\
\hline $\begin{array}{l}\text { Change in cost (over two } \\
\text { years) }\end{array}$ & $-16.22(118.00)$ & $-17.62(102.74)$ & $-9.17(94.37)$ & $-31.07(136.17)$ \\
\hline Visit costs (over two years) & $631.86(324.41)$ & $753.19(246.01)$ & $223.83(115.10)$ & $0.00(0.00)$ \\
\hline Overall change in cost & $615.64(323.20)$ & $735.57(260.13)$ & $214.66(128.02)$ & $-31.07(129.78)$ \\
\hline \multicolumn{5}{|l|}{ EQ-5D score: } \\
\hline Baseline & $0.542(0.274)$ & $0.531(0.305)$ & $0.587(0.245)$ & $0.555(0.281)$ \\
\hline 6 month & $0.643(0.211)$ & $0.603(0.275)$ & $0.649(0.254)$ & $0.591(0.251)$ \\
\hline 12 month & $0.608(0.255)$ & $0.612(0.267)$ & $0.626(0.250)$ & $0.617(0.260)$ \\
\hline 24 month & $0.642(0.245)$ & $0.609(0.279)$ & $0.649(0.260)$ & $0.601(0.266)$ \\
\hline QALY gain & $0.147(0.340)$ & $0.133(0.415)$ & $0.090(0.388)$ & $0.085(0.349)$ \\
\hline Incremental cost ( $v$ leaflet) & 646.71 & 766.64 & 245.73 & - \\
\hline Incremental effect ( $v$ leaflet) & 0.062 & 0.048 & 0.005 & - \\
\hline ICER & 10649 & Dominated & ED & - \\
\hline
\end{tabular}




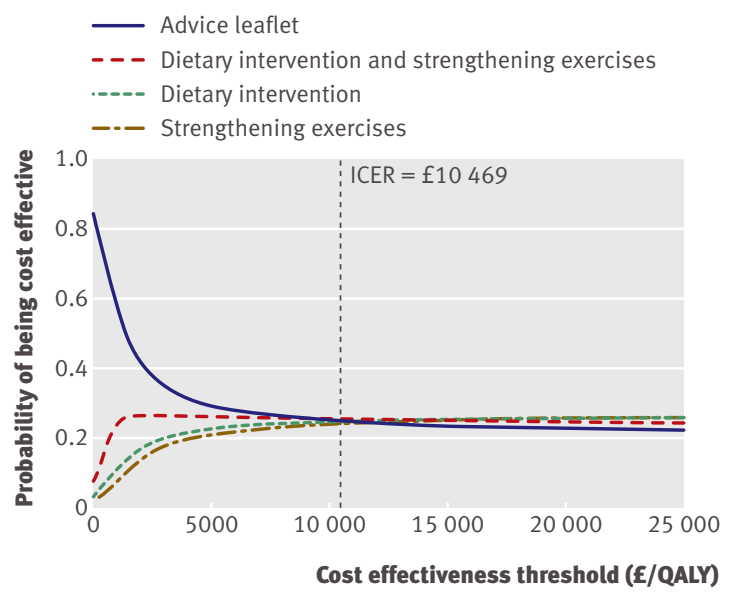

Decision uncertainty: plots of cost effectiveness acceptability curves for four intervention groups (ICER=incremental cost effectiveness ratio)

the four intervention groups, estimated analgesic costs were lower at follow-up than at baseline (table 1). We combined the estimates of costs for visits and analgesics to estimate the overall change in cost to the health service: this ranged between an increase in mean cost of $£ 735.57$ (for the dietary intervention) and a decrease in mean cost of $£ 31.07$ (for the leaflet) (table 1).

To determine use of other health services, one year after randomisation we contacted 188 (82\%) of the proposed subsample of 229 participants. There was no significant difference across each of the four intervention groups in the number of knee pain related visits to the general practitioner, nurse, other healthcare professionals, or hospital in the preceding four weeks (table 2).

\section{Outcomes}

At baseline, six, 12, and 24 months an average of 336 participants $(86 \%)$ completed the EQ-5D. Table 1 shows the mean scores at each of these time points (after imputation) for the four interventions groups, along with the mean QALY gain (over the two year trial period). Leaflet provision was associated with the lowest gain (0.085 QALYs), and dietary intervention plus quadriceps strengthening exercises was associated with the largest gain (0.147 QALYs).

\section{Cost effectiveness}

Comparison of the mean change in cost and mean QALY gain (table 1) across the four intervention groups showed that dietary intervention alone was dominated by dietary intervention plus quadriceps strengthening exercises. Similarly, quadriceps strengthening exercises was not cost effective as it was subject to extended dominance (combinations of leaflet provision and dietary intervention plus quadriceps strengthening exercises could provide a higher benefit at equivalent cost). Compared with leaflet provision, dietary intervention plus quadriceps strengthening exercises was both more costly (mean incremental cost $£ 646.71,95 \%$ confidence interval $£ 578.15$ to $£ 709.62$ ) and more effective (mean incremental effect $0.062,-0.035$ to 0.167 ), with an estimated incremental cost per QALY gain (incremental cost effectiveness ratio) of $£ 10469.44$ ( $\$ 3738.28$ to dominated) (table 1). Thus, dietary intervention plus quadriceps strengthening exercises is likely to be deemed cost effective as it has an incremental cost effectiveness ratio that is more favourable than a threshold of $£ 20000$ - $£ 30000$ per QALY.

\section{Decision uncertainty}

The cost effectiveness acceptability curves indicate that for threshold values $\geq £ 5000$ per QALY the probability of cost effectiveness was $<30 \%$ for all four interventions, showing that there is a large level of uncertainty associated with the decision as to which intervention is the most cost effective (figure). The probability that dietary intervention plus quadriceps strengthening exercises was the most cost effective intervention was $23.1 \%$ at $\lambda=£ 20000$ per QALY.

\section{Sensitivity analysis}

When we assumed that the unit cost of a visit for those in the leaflet arm of the trial was equivalent to that in the quadriceps strengthening exercises arm (£44.60), the estimated mean cost per participant in the leaflet arm was $£ 235.83$. In this situation leaflet provision would have been dominated by quadriceps strengthening exercises. The incremental cost effectiveness ratio for dietary intervention plus quadriceps strengthening exercises would thereby have been estimated to be more favourable at $£ 6148.59$ compared with quadriceps strengthening exercises.

\section{DISCUSSION}

In this randomised controlled trial of four interventions for knee pain in overweight and obese adults, dietary intervention plus quadriceps strengthening exercises was associated with the highest mean gain

Table 2 | Mean number of reported visits related to knee pain in preceding four weeks at one year after randomisation within each of intervention group

\begin{tabular}{lccccc} 
& $\begin{array}{c}\text { Dietary intervention plus } \\
\text { strengthening exercises }\end{array}$ & $\begin{array}{c}\text { Dietary } \\
\text { intervention }\end{array}$ & $\begin{array}{c}\text { Strengthening } \\
\text { exercises }\end{array}$ & Leaflet provision & F statistic \\
General practitioner & 0.14 & 0.18 & 0.19 & 0.15 & 0.104 \\
\hline Nurse & 0.39 & 0.47 & 0.47 & 0.41 & 0.105 \\
\hline Other healthcare professionals & 0.23 & 0.30 & 0.09 & 0.931 & 0.28 \\
\hline Outpatient visits & 0.36 & 0.28 & 0.50 & 0.606 \\
\hline Inpatient admissions & 0.02 & 0.00 & 0.00 & 0.660 & 0.02 \\
\hline
\end{tabular}




WHAT IS ALREADY KNOWN ON THIS TOPIC
The prevalence of knee pain increases with age and for
those who are overweight
No assessment as to the cost effectiveness of dietary
intervention or quadriceps strengthening exercises, or both,
has been made for overweight individuals
WHAT THIS STUDY ADDS
Provision of dietary intervention plus quadriceps
strengthening exercises was estimated to be cost effective
at a threshold of $£ 20000$ per QALY, though there was a large
level of associated uncertainty

in QALYs. It was also, however, more costly, with an estimated incremental cost effectiveness ratio of $£ 10469$. As this value falls below a threshold value of $£ 20000-£ 30000$ per QALY, the provision of dietary intervention plus quadriceps strengthening exercises represents a cost effective use of scarce healthcare resources. The probability that this was the most cost effective intervention, however, was only $23.1 \%$ when $\lambda=£ 20000 /$ QALY.

\section{Strengths and weaknesses}

Though our two year follow-up period was longer than in many other clinical trials, we did not estimate the long term costs and benefits of these interventions. Furthermore, as we did not systematically monitor compliance with either the dietary plan or exercise advice, our ability to predict the long term effects of these interventions is diminished. That said, were the benefits of providing dietary intervention plus quadriceps strengthening exercises to be sustained beyond the two year follow-up period then it is likely that, had the analysis taken a lifetime perspective, the estimates as to the cost effectiveness of this intervention would have been more favourable. Similarly, within this study we did not evaluate the provision of group exercise programmes, which others have shown to be effective, ${ }^{3}$ and it might be that, because of economies of scale, it is more cost effective to use group based exercise classes rather than undertake one to one healthcare professional visits.

\section{Comparison to other studies}

We are aware of two studies that have estimated the cost effectiveness of different interventions for people with knee pain (in non-overweight populations), ${ }^{23}$ though only one of these studies measured effectiveness in terms of utility. ${ }^{3}$ Our results are in contrast with those of Hurley et al, who found that the provision of a rehabilitation programme by a physiotherapist (in addition to usual care) was less effective, in terms of QALYs gained, than usual primary care. ${ }^{3}$ Our results are more in line with those studies that have used WOMAC to measure effectiveness. Thomas et al found that, compared with either telephone contact or no intervention, an exercise programme was estimated to be more effective according to WOMAC but also more costly and was not associated with a reduction in other health costs. ${ }^{2}$ Hurley et al found that, compared with usual care, a greater proportion of those who received rehabilitation by a physiotherapist had a clinically meaningful improvement according to WOMAC but that this was also more costly. ${ }^{3}$

\section{Explanations}

The finding that dietary intervention plus quadriceps strengthening exercises was estimated to be cost effective aligns with the findings of the accompanying clinical paper, ${ }^{14}$ in which strengthening exercises were associated with a reduction in pain according to WOMAC and that both dietary intervention and strengthening exercises improved functioning according to WOMAC. Additionally, the high level of uncertainty associated with the estimates of cost effectiveness can be partially explained by the fact that less than two thirds of those in the dietary intervention plus quadriceps strengthening exercises group actually received a net QALY gain over the two year period.

\section{Implications and future research}

The provision of dietary intervention plus quadriceps strengthening exercises to individuals aged $\geq 45$ with a $\mathrm{BMI} \geq 28$ is likely to represent a cost effective use of scarce NHS resources. There was, however, a large level of uncertainty associated with this decision, which was partially accounted for by the fact that there was a high level of variation in the benefits that different individuals received. Further research might therefore focus on the value of personalised knee pain interventions; some participants might feel they need a high level of support, whereas others, after initial instruction, might be quite able to adhere to the intervention without further support. Here, one might also consider a more thorough investigation of the impact that these interventions have on the number of visits to other healthcare professionals related to knee pain. Though we found no significant difference in this variable in a subsample of patients at a single time point (see table 2), it is conceivable that such services might be affected as it has previously been shown that patients with knee pain who received an exercise programme had both significantly fewer general practice consultations and used less pain relief than those who received only an advice leaflet. ${ }^{13}$

Contributors: KRM was principal investigator. All authors contributed towards the development of the economic evaluation protocol. GRB and THS analysed the economic data, wrote the original draft, and are guarantors. All authors commented on the draft and the interpretation of the findings.

Funding: The study was funded by the UK Arthritis Research Campaign (ARC) (grant No 13550). ARC had no role in study design; in the collection, analysis, and interpretation of data; in the writing of the report; or in the decision to submit the paper for publication.

Competing interests: None declared.

Ethical approval: This study was approved by the Nottingham research ethics committee (REC Q1090219); all participants gave informed consent. 
1 Jinks C, Jordan K, Ong BN, Croft P. A brief screening tool for knee pain in primary care (KNEST). 2. Results from a survey in the general population aged 50 and over. Rheumatology 2004;43:55-61.

2 Thomas KS, Miller P, Doherty M, Muir KR, Jones AC, O'Reilly SC. Cos effectiveness of a two-year home exercise program for the treatment of knee pain. Arthritis Rheum 2005;53:388-94.

3 Hurley MV, Walsh NE, Mitchell HL, Pimm TJ, Williamson E, Jones RH, et al. Economic evaluation of a rehabilitation program integrating exercise, self-management, and active coping strategies for chronic knee pain. Arthritis Rheum 2007;57:1220-9.

4 Porcheret $M$, Jordan $K$, Jinks C, Croft P, in collaboration with the Primary Care Rheumatology Society. Primary care treatment of knee pain-a survey in older adults. Rheumatology 2007;1694-1700.

5 Porcheret $\mathrm{M}$, Jordan $\mathrm{K}$, Croft P, in collaboration with the Primary Care Rheumatology Society. Treatment of knee pain in older adults in primary care: development of an evidence-based model of care. Rheumatology 2007;46:638-48.

6 Messier SP, Loeser RF, Miller GD, Morgan TM, Rejeski WJ, Sevick MA, et al. Exercise and dietary weight loss in overweight and obese older adults with knee osteoarthritis: the arthritis, diet, and activity promotion trial. Arthritis Rheum 2004;50:1501-10.

7 Nevitt MC, Lane N. Body weight and osteoarthritis. Am J Med 1999;107:632-3.

8 Thomas KS, Muir KR, Doherty M, Jones AC, O’Reilly SC, Bassey EJ. Home based exercise programme for knee pain and knee osteoarthritis: randomised controlled trial. BMJ 2002;325:752-5.

9 Felson DT, Zhang Y. An update on the epidemiology of knee and hip osteoarthritis with a view to prevention. Arthritis Rheum 1998;41:1343-55.

10 O’Reilly SC, Jones A, Muir KR, Doherty M. Quadriceps weakness in knee osteoarthritis: the effect on pain and disability. Ann Rheum Dis 1998;56:588-94.

11 Slemenda C, Brandt KD, Heilmam DK, Mazzuca SA, Braunsteing EM, Katz BP, et al. Quadriceps weakness and osteoarthritis of the knee. Ann Intern Med 1997;127:97-104.

12 O'Reilly SC, Muir KR, Doherty M. Effectiveness of home exercise on pain and disability from osteoarthritis of the knee: a randomised controlled trial. Ann Rheum Dis 1999;58:15-9.

13 Hay EM, Foster NE, Thomas E, Peat G, Phelan M, Yates HE, et al. Effectiveness of community physiotherapy and enhanced pharmacy review for knee pain in people aged over 55 presenting to primary care: pragmatic randomised trial. BMJ 2006;333:995.

14 Jenkinson CM, Doherty M, Avery AJ, Read A, Taylor M, Sach TH, et al. Effects of dietary intervention and quadriceps strengthening exercises on pain and function in overweight people with knee pain: randomised controlled trial. BMJ 2009 doi:10.1136/bmj.b3170.

15 HM Treasury. The green book appraisal and evaluation in central government. London: Stationery Office, 2003.

16 Curtis L. Unit costs of health and social care. Canterbury: University of Kent: Personal Social Services Research Unit, 2007.

17 Prescribing Support Unit. Prescription cost analysis: England 2006. London: Information Centre, Government Statistical Service, 2007.

18 Schafer JL. Multiple imputation: a primer. Stat Methods Med Res 1999;8:3-15.

19 Manca A, Hawkins N, Sculpher MJ. Estimating mean QALYs in trialbased cost-effectiveness analysis: the importance of controlling for baseline utility. Health Economics 2005;14:487-96.

20 Bellamy N, Buchanan WW, Goldsmith CH, Campbell J, Stitt LW. Validation study of WOMAC: a health status instrument for measuring clinically important patient relevant outcomes to antirheumatic drug therapy in patients with osteoarthritis of the hip or knee. J Rheumatol 1988;15:1833-40.

21 Brooks R. EuroQol: the current state of play. Health Policy 1996;37:53-72.

22 Drummond MF, Sculpher MJ, Torrance GW, O’Brien BJ, Stoddart GL. Methods for the economic evaluation of health care programmes. $3 \mathrm{rd}$ ed. New York: Oxford University Press, 2005.

23 Dolan P. Modelling valuations for EuroQol health states. Med Care 1997;35:1095-108.

24 Cantor SB. Cost-effectiveness analysis, extended dominance, and ethics: a quantitative assessment. Med Decis Making 1994;14:259-65.

25 Briggs $\mathrm{AH}$, Wonderling DE, Mooney CZ. Pulling cost-effectiveness analysis up by its bootstraps: a non-parametric approach to confidence interval estimation. Health Econ 1997;6:327-40.

26 National Institute for Health and Clinical Excellence. Guide to the methods of technology appraisal. London: NICE, 2008.

27 Fenwick E, Claxton K, Sculpher MJ. Representing uncertainty: the rol of cost-effectiveness acceptability curves. Health Econ 2001;10:779-87.

28 Barton GR, Briggs AH, Fenwick EA. Optimal cost-effective decisions: the role of the cost-effectiveness acceptability curve (CEAC), costeffectiveness acceptability frontier (CEAF) and expected value of perfect information (EVPI). Value Health 2008;11:886-97.

Accepted: 12 December 2008 